\title{
On the Laplacian Coefficients of Bicyclic Graphs
}

\author{
Xinying Pai \\ College of Science, China University of Petroleum, China
}

Copyright (C) 2015 by authors, all rights reserved. Authors agree that this article remains permanently open access under the terms of the Creative Commons Attribution License 4.0 International License

\begin{abstract}
In this paper, we investigate how the Laplacian coefficients changed after some graph transformations. So, I express some results about Laplacian coefficients ordering of graphs, focusing our attention to the bicyclic graphs. Finally, as an application of these results, we discuss the ordering of graphs based on their Laplacian like energy.
\end{abstract}

Keywords Laplacian Coefficients, Bicyclic Graph, Laplacian-like Energy

\section{Introduction}

Let $G=(V, E)$ be a simple undirected graph with $n$ vertices, $|E|$ edges and $L(G)=D(G)-A(G)$ be its Laplacian matrix. The Laplacian polynomial $\Phi(G, \lambda)$ of $G$ is the characteristic polynomial of its Laplacian matrix. That is

$$
\Phi(G, \lambda)=\operatorname{det}\left(\lambda I_{n}-L(G)\right)=\sum_{k=0}^{n}(-1)^{k} c_{k}(G) \lambda^{n-k} .
$$

It is well-known that $c_{0}(G)=1, c_{1}(G)=2|E|$, $c_{n}(G)=0$, and $c_{n-1}(G)=n \tau(G)$, where $\tau(G)$ is the number of spanning trees of $G$ (see [1]).
Recently, the study on the Laplacian coefficient attracts much attention. Some works on Laplacian coefficients can be found in [2-8]. In this paper, we determine the largest coefficient among all the bicyclic graphs of order $n$.

Bicyclic graphs are connected graphs in which the number of edges equals the number of vertices plus one. Let $B_{n}$ be the set of all connected bicyclic graphs of order $n$. Obviously $B_{n}$ consists of two types of graphs: one type, denoted by $B_{n}^{\infty}$, are those graphs each of which is an $\infty$ -graph with trees attached; the other type, denoted by $B_{n}^{\theta}$, are those graphs each of which is an $\theta$-graph with trees attached. Then $B_{n}=B_{n}^{\infty} \cup B_{n}^{\theta}$.

\section{Transformations and Lemmas}

Let $F$ be a spanning forest of $G$ with components $T_{i}, i=1,2, \ldots, k$ having $n_{i}$ vertices, and set $\gamma(F)=\prod_{i=1}^{k} n_{i}$. The Laplacian coefficients $c_{k}(G)$ of a graph $G$ can be expressed in terms of subtree structures of $G$ by the following result of Kelmans and Chelnokov [6].
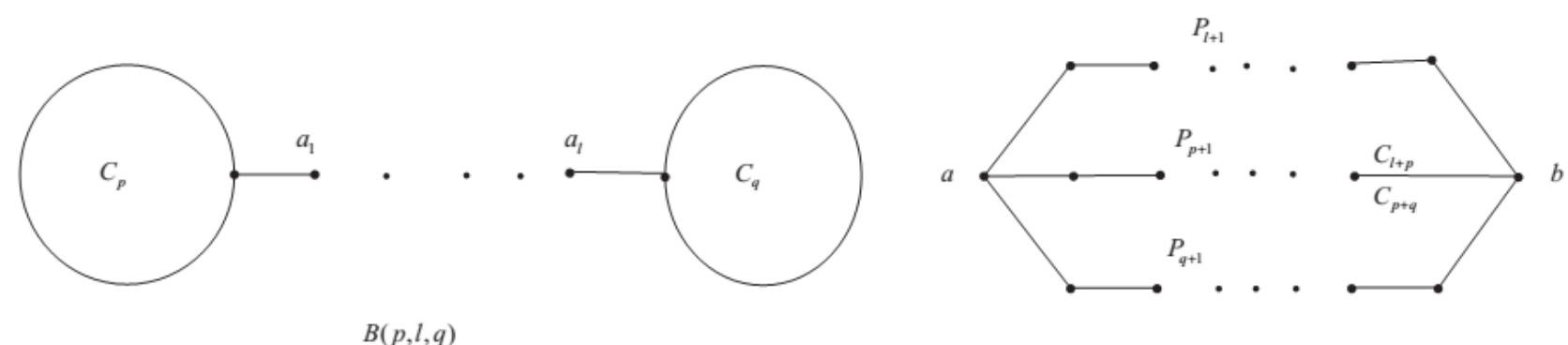

$B(p, l, q)$

$P(l, p, q)$

Figure 1. $\mathrm{B}(p, l, q)$ and $P(l, p, q)$ 
Lemma 2.1([6]) The Laplacian coefficient $c_{n-k}(G)$ of a graph $G$ is given by $c_{n-k}(G)=\sum_{F \in F_{k}} \gamma(F)$, where $F_{k}$ is the set of all spanning forests of $G$ with exactly $k$ components.

Let $N_{G}(v)$ denote the adjacent vertex set of a vertex $v$ of a graph $G$ and let $d(v)$ denote the degree of $v$ in $G$. Let $C_{p}$ denote the cycle on $n$ vertices and let $d_{C_{p}}(u, v)$ denote the distance between $u, v$ on the cycle $C_{p}$.

Definition 2.2([3]) Let $w$ be a vertex of $G$ with degree $d(w) \geq 3$ and $P=w v_{1} v_{2} \cdots v_{p}$ and $Q=w u_{1} u_{2} \cdots u_{q}$ are distinct pendant paths of lengths $p \geq 1$ and $q \geq 1$, respectively, attached at $w$. Let $G^{\prime}=G-w u_{1}+v_{p} u_{1} \triangleq$ $\pi(G, w, P, Q)$. We say that $G^{\prime}$ is a $\pi$-transformation of $G$.

Lemma 2.3([3]) Let $G^{\prime}=\pi(G, w, P, Q)$. Then for every $k=0,1, \ldots, n, \quad c_{k}(G) \leq c_{k}\left(G^{\prime}\right)$, with equality if and only if $k \in\{0,1, n-1, n\}$.

Definition 2.4([3]) Let $w$ be a vertex in a cycle $C$ of a unicyclic graph $G$. Assume that $G$ has pendant path $P=w v_{1} v_{2} \cdots v_{p}$ attached to $w$. If $u$ is one of two neighbors of $w$ in $C$, let $G^{\prime}=\gamma(G, w, P)$ be the graph obtained from $G$ by removing edge $u w$ and adding edge $u v_{p}$. We say that $G^{\prime}$ is a $\gamma$-transform of $G$.

Lemma 2.5([3]) Let $G^{\prime}=\gamma(G, w, P)$. For $0 \leq k \leq n$ holds $c_{k}(G) \leq c_{k}\left(G^{\prime}\right)$, with equality if and only if $k \in\{0,1, n\}$.

Definition 2.6([9]) Let $v$ be a vertex of a tree $T$ of degree $m+1$. Suppose that $P_{1}, P_{2}, \ldots, P_{m}$ are pendent paths incident with $v$, with lengths $n_{i} \geq 1, i=1,2, \ldots, m$. Let $w$ be the neighbor of $v$ distinct from the starting vertices of paths $v_{1}, v_{2}, \ldots, v_{m}$, respectively. We form a tree $T^{\prime}=\delta(T, v)$ by removing the edges $v v_{1}, v v_{2}, \ldots, v v_{m-1}$ from $T$ and adding $m-1$ new edges $w v_{1}, w v_{2}, \ldots, w v_{m-1}$ incident with $w$. We say that $T^{\prime}$ is a $\delta$-transform of $T$.

Lemma 2.7([9]) Let $T$ be an arbitrary tree, rooted at the center vertex. Let vertex $v$ be on the deepest level of tree $T$ among all branching vertices with degree at least three. Then for the $\delta$-transformation tree $T^{\prime}=\delta(T, v)$ and $0 \leq k \leq n$ holds $c_{k}(T) \geq c_{k}\left(T^{\prime}\right)$.

Definition 2.8([11]) Let $G$ be a simple connected graph with $n$ vertices, and let $P_{s}=w_{1} w_{2} \cdots w_{s}$ be a path of $G$ not contained in cycles of lengths $s$ and $s+1$ such that $s \geq 2, d_{G}\left(w_{1}\right) \geq 2, d_{G}\left(w_{2}\right)=\cdots=d_{G}\left(w_{s-1}\right)=2, d_{G}\left(w_{s}\right) \geq 2$, where $d_{G}(u)$ is the degree of $u$ in $G$. Let $N_{G}\left(w_{1}\right)=\left\{w_{2}, u_{1}, u_{2}, \ldots, u_{p}\right\}$ be the set of vertices adjacent to $w_{1}$ in $G$, and put

$G_{P_{s}}=G-\left\{w_{1} u_{1}, w_{1} u_{2}, \ldots, w_{1} u_{p}\right\}+\left\{w_{s} u_{1}, w_{s} u_{2}, \ldots, w_{s} u_{p}\right\}$. The graph $G_{P_{s}}$ is called a $\chi_{s}$-transform of the graph G.

Lemma 2.9([11]) Let $G$ and $G_{P_{s}}$ be the $n$-vertex graphs given in Definition 2.8. Then $c_{k}(G) \geq c_{k}\left(G_{P_{s}}\right), k=0,1, \ldots, n$, with equality if and only if $k \in\{0,1, n-1, n\}$ if $P_{s}$ is not contained in any cycle of $G$ or $k \in\{0,1, n\}$ otherwise.

Lemma 2.10([10]) Let $G$ be an acyclic graph on $n$ vertices, then $c_{k}(G)=m(S(G), k)$ for $0 \leq k \leq n$.

If $v$ is a pendant vertex of the graph $G$, adjacent to the vertex $u$, then the matching numbers of $G$ conform to the recurrence relation

$$
m(G, k)=m(G-v, k)+m(G-u-v, k-1) .
$$

Definition 2.11 Let $C_{p}=u_{1} u_{2} \ldots u_{p-1} v_{1} u_{1}$ be a cycle of a connected unicyclic graph $G$. Assume that $G$ has pendant paths $P=v_{l} w_{1} w_{2} \cdots w_{s}$ and $Q$ attached to $v_{l}$. Let $G_{\gamma}=G-u_{1} v_{1}+u_{1} w_{r} \triangleq \gamma\left(G, v_{1}, w_{r}\right)$, where $1 \leq r \leq s$. We say that $G_{\gamma}$ is a $\gamma$-transform of $G$ (see Fig. 2).

Lemma 2.12 Let $G$ and $G_{\gamma}$ be the unicyclic graphs as shown in Fig. 2, $l, r$ and $s$ are positive integers. Suppose that $1 \leq r \leq s$. Then for every $k=0,1, \ldots, n$, $c_{k}(G) \leq c_{k}\left(G_{\gamma}\right)$, with equality if and only if $k \in\{0,1, n\}$.

\section{Proof.}

$c_{0}(G)=c_{0}\left(G_{\gamma}\right)=1, c_{1}(G)=c_{1}\left(G_{\gamma}\right)=$ $=2|E|, c_{n}(G)=c_{n}\left(G_{\gamma}\right)=0, c_{n-1}(G)<c_{n-1}\left(G_{\gamma}\right)$.

Let $F_{k}$ and $F_{k}^{\gamma}$ be the sets of spanning forests of $G$ and $G_{\gamma}$ with exactly $k$ components, respectively. We distinguish the following two cases.

Case 1. $l=1$. According to the definition of the spanning forest, the all edges in the cycle $C_{p}$ can't exist in any forest $F$ at the same time, where $F$ is the arbitrary forests in $F_{k}$. We distinguish the following two cases.

Case 1.1. If we remove the edge $u_{1} v_{1}$ in $G$ and remove the edge $u_{1} w_{r}$ in $G_{\gamma}$, we can get the trees $T$ and $T_{\gamma}$. It is easy to see that $T=T_{\gamma}$. 

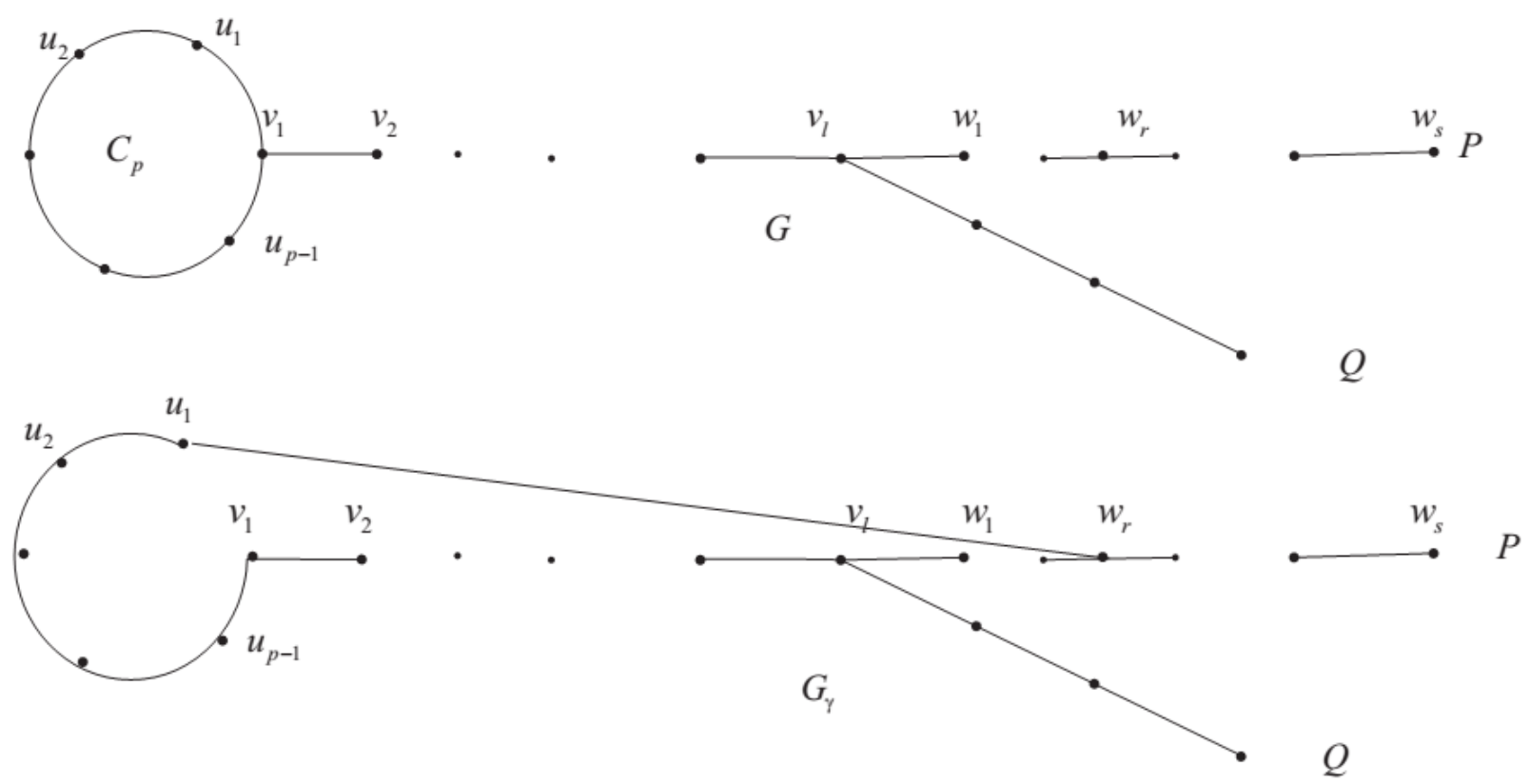

Figure 2. $G$ and $G_{\gamma}$

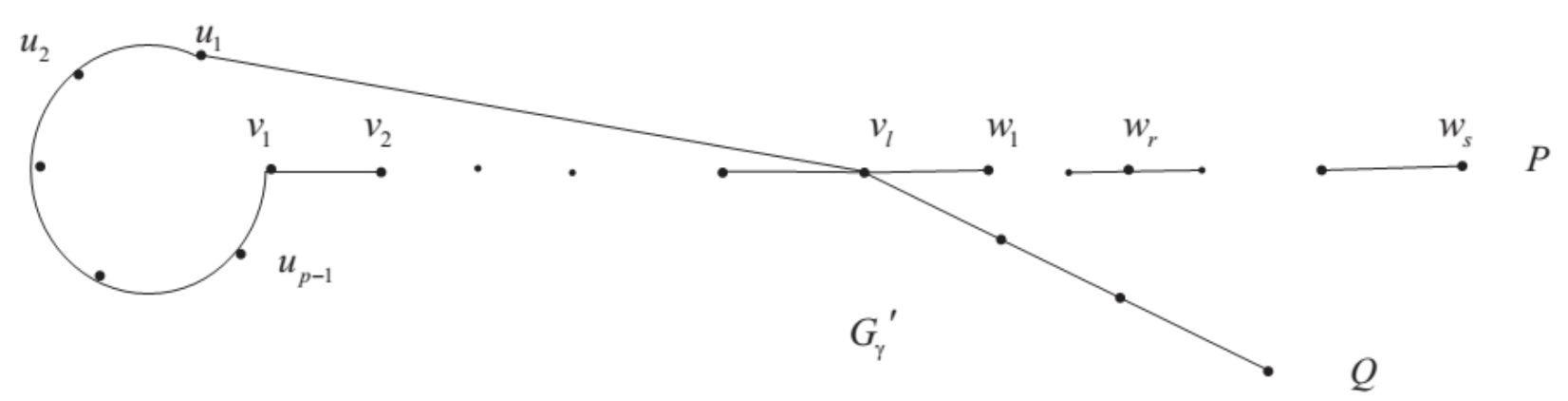

Figure 3. $\quad G_{\gamma}^{\prime}$

Case 1.2. If we remove the edges $u_{i} u_{i+1}(i=1,2, \cdots, p-2)$, and $u_{p-1} v_{1}$ in $G$ and $G_{\gamma}$, we can get the trees $T_{i}$ and $T_{i}^{\gamma} \quad(i=1,2, \cdots, p-1)$, respectively. According to Definition 2.6, we know that $T_{i}=\delta\left(T_{i}^{\gamma}, w_{r}\right)(i=1,2, \cdots, p-1)$, by Lemma 2.7 , it is easy to see $c_{k}\left(T_{i}\right) \leq c_{k}\left(T_{i}^{\gamma}\right)$. At the same time, we can get the number of trees obtained in $G_{\gamma}$ more than that of trees obtained in $G$. By Lemma 2.1, it is easy to see that for every $k=0,1, \ldots, n, \quad c_{k}(G) \leq c_{k}\left(G_{\gamma}\right)$, with equality if and only if $k \in\{0,1, n\}$.

Case 2. $l>1$. Let $G_{\gamma}^{\prime}=G_{\gamma}-u_{1} w_{r}+u_{1} v_{l}$ (see Fig. 3), it is easy to see that the relationship between $G_{\gamma}^{\prime}$ and $G_{\gamma}$ is similar to the relationship between $G$ and $G_{\gamma}$ in Case 1 . According to the proof of Case 1, we have that for every $k=0,1, \ldots, n, \quad c_{k}\left(G_{\gamma}^{\prime}\right) \leq c_{k}\left(G_{\gamma}\right)$, with equality if and only if $k \in\{0,1, n\}$. By Definition 2.8, $G$ can be obtained by $G_{\gamma}^{\prime}$ through $\chi_{s}-$ transform. According to Lemma 2.9 , we can get that for every $k=0,1, \ldots, n$, $c_{k}(G) \leq c_{k}\left(G_{\gamma}^{\prime}\right)$, with equality if and only if $k \in\{0,1, n\}$. From the above, we have that for every $k=0,1, \ldots, n, c_{k}(G) \leq c_{k}\left(G_{\gamma}\right)$, with equality if and only if $k \in\{0,1, n\}$. Then the conclusion of Lemma 2.12 holds.

\section{Main Results}

\subsection{Laplacian Coefficients of Bicyclic Graphs}

Definition 3.1 Let $w$ be a vertex of the cycle $C_{p}$ (or $\left.C_{q}, C_{l+p}, C_{p+q}\right)$ in a connected bicyclic graph $G$. Assume that $G$ has a pendant path $P=w z_{1} z_{2} \cdots z_{r}$ attached at $w$. If $u$ is one of two neighbors adjacent to $w$ in $C_{p}$ (or $\left.C_{q}, C_{l+p}, C_{p+q}\right)$. Then $G_{1}=\eta_{1}(G, w, P)$ 
is a graph obtained from $G$ by removing edge $u w$ and adding edge $u z_{r}$. We say that $G_{1}$ is a $\eta_{1}$-transformation of $G$.

Theorem 3.2 Let $G$ be a bicyclic graph with $n$ vertices, $G_{1}=\eta_{1}(G, w, P)$. Then for every $k=0,1, \ldots, n$, $c_{k}(G) \leq c_{k}\left(G_{1}\right)$, with equality if and only if $k \in\{0,1, n\}$.

Proof. Without loss of generality, we assume that $w$ is a vertex of the cycle $C_{p}$.

$c_{0}(G)=c_{0}\left(G_{1}\right)=1, c_{1}(G)=c_{1}\left(G_{1}\right)=2|E|, c_{n}(G)=$ $=c_{n}\left(G_{1}\right)=0, c_{n-1}(G)<c_{n-1}\left(G_{1}\right)$.

Let $F_{k}$ and $F_{k}^{1}$ be the sets of spanning forests of $G$ and $G_{1}$ with exactly $k$ components, respectively. If we remove one of the edges in $C_{q}$, say $u v$, we can get the unicyclic graphs $U$ and $U^{\prime}$ in $G$ and $G_{1}$. By Definition 2.4 and Lemma 2.5, we have $U^{\prime}=\gamma(U, w, P)$ and for every $0 \leq k \leq n$ holds $c_{k}(U) \leq c_{k}\left(U^{\prime}\right)$, with equality if and only if $k \in\{0,1, n\}$. At the same time, we can get the same number of unicyclic graphs obtained from $G$ and $G_{1}$. According to Lemma 2.1, it is easy to see that for every $k=0,1, \ldots, n, \quad c_{k}(G) \leq c_{k}\left(G_{1}\right)$, with equality if and only if $k \in\{0,1, n\}$. Then the conclusion of Theorem 3.2 holds.

Let $\mathrm{B}(p, l, q)=\{B(p, l, q): p \geq 3, q \geq 3, l \geq 1\}$ and $\mathrm{P}(l, p, q)=\{P(l, p, q): l, p, q \geq 1$ and at most one of them is 1$\}$. Denote $B(n)=\mathrm{B}(p, l, q) \cup \mathrm{P}(l, p, q)$.

Theorem 3.3 Let $G$ be a bicyclic graph. For $k=0,1, \ldots, n$, there exists some graph $H \in B(n)$ to satisfy the following inequality $c_{k}(G) \leq c_{k}(H)$.

Proof. Let $T_{i}$ be the tree attached at $u_{i}$. We can use Definition 2.2 to the vertex of $T_{i}$ which is the largest distance from the root $u_{i}$, as long as $T_{i}$ is not a path. By using Lemma 2.3, we increase Laplacian coefficients of $G$.

Assume that $G \in B_{n}^{\infty}$ has pendant path $P=a_{i} z_{1} z_{2} \cdots z_{r}$ attached at $a_{i},(2 \leq i \leq l-1)$. Let $G_{1}$ be the graph obtained from $G$ by removing the edge $a_{i} a_{i+1}$ and adding the edge $a_{i+1} z_{r}$. Obviously, we can have

$$
\begin{aligned}
& c_{0}(G)=c_{0}(H)=1, c_{1}(G)=c_{1}(H)=2|E|, c_{n}(G)= \\
& =c_{n}(H)=0, c_{n-1}(G)=c_{n-1}(H) .
\end{aligned}
$$

Let $F_{k}$ and $F_{k}^{H}$ be the sets of spanning forests of $G$ and $H$ with exactly $k$ components, respectively. Similar to the above prove, we can get the tree $T\left(T^{\prime}\right)$ on $G(H)$. By Lemma 2.10 and (2.1), it is easy to see that for every $0 \leq k \leq n$ holds $c_{k}(T) \leq c_{k}\left(T^{\prime}\right)$, with equality if and only if $k \in\{0,1, n-1, n\}$. We can get the same number of trees obtained from $G$ and $H$. According to Lemma 2.1, it is easy to see that for every $k=0,1, \ldots, n$, $c_{k}(G) \leq c_{k}(H)$, with equality if and only if $k \in\{0,1, n\}$.

Finally, we can apply $\eta_{1}$-transformation to the graph as long as it is not in $B(n)$. According to Theorem 3.2 and the above proof, we further increase its Laplacian coefficients. This means that the maximum Laplacian coefficients must be obtained from $B(n)$.

Definition 3.4 Let $G \in \mathrm{B}(p, l, q)$ and $p \geq 3, q \geq 3, l \geq 1$. Suppose that $u \in N_{C_{p}}\left(a_{1}\right)$, $v \in V\left(C_{q}\right)$ and $d_{C_{q}}\left(a_{l}, v\right)=\left\lfloor\frac{q}{2}\right\rfloor$. Then $G_{2}=\eta_{2}(G, u, v)$ is a graph obtained by removing edge $u a_{1}$ and adding edge $u v$. We say that $G_{2}$ is a $\eta_{2}$ -transformation\} of $G$ (see Fig. 4, if $l=1$, see Fig. 5).

Theorem 3.5 Let $G \in \mathrm{B}(p, l, q)$ and $p \geq 3, q \geq 3, l \geq 1$. If $G_{2}=\eta_{2}(G, u, v)$, then for every $k=0,1, \ldots, n$, we have $c_{k}(G) \leq c_{k}\left(G_{2}\right)$.

Proof. Obviously, $c_{0}(G)=c_{0}\left(G_{2}\right)=1, c_{1}(G)=c_{1}\left(G_{2}\right)=$. If $=2|E|, c_{n}(G)=c_{n}\left(G_{2}\right)=0$

$q$ is an even number,

\section{Main Results}

\subsection{Laplacian Coefficients of Bicyclic Graphs}

Definition 3.1 Let $w$ be a vertex of the cycle $C_{p}$ (or $C_{q}, C_{l+p}, C_{p+q}$ ) in a connected bicyclic graph $G$. Assume that $G$ has a pendant path $P=w z_{1} z_{2} \cdots z_{r}$ attached at $w$. If $u$ is one of two neighbors adjacent to $w$ in $C_{p}$ (or $C_{q}, C_{l+p}, C_{p+q}$ ). Then $G_{1}=\eta_{1}(G, w, P)$ is a graph obtained from $G$ by removing edge $u w$ and adding edge $u z_{r}$. We say that $G_{1}$ is a $\eta_{1}$-transformation of $G$.

Theorem 3.2 Let $G$ be a bicyclic graph with $n$ vertices, $G_{1}=\eta_{1}(G, w, P)$. Then for every $k=0,1, \ldots, n$, $c_{k}(G) \leq c_{k}\left(G_{1}\right)$, with equality if and only if $k \in\{0,1, n\}$. 
Proof. Without loss of generality, we assume that $w$ is a vertex of the cycle $C_{p}$.

$c_{0}(G)=c_{0}\left(G_{1}\right)=1, c_{1}(G)=c_{1}\left(G_{1}\right)=$ $=2|E|, c_{n}(G)=c_{n}\left(G_{1}\right)=0, c_{n-1}(G)<c_{n-1}\left(G_{1}\right)$.

Let $F_{k}$ and $F_{k}^{1}$ be the sets of spanning forests of $G$ and $G_{1}$ with exactly $k$ components, respectively. If we remove one of the edges in $C_{q}$, say $u v$, we can get the unicyclic graphs $U$ and $U^{\prime}$ in $G$ and $G_{1}$. By Definition 2.4 and Lemma 2.5, we have $U^{\prime}=\gamma(U, w, P)$ and for every $0 \leq k \leq n$ holds $c_{k}(U) \leq c_{k}\left(U^{\prime}\right)$, with equality if and only if $k \in\{0,1, n\}$. At the same time, we can get the same number of unicyclic graphs obtained from $G$ and $G_{1}$. According to Lemma 2.1, it is easy to see that for every $k=0,1, \ldots, n, \quad c_{k}(G) \leq c_{k}\left(G_{1}\right)$, with equality if and only if $k \in\{0,1, n\}$. Then the conclusion of Theorem 3.2 holds.

Let $\mathrm{B}(p, l, q)=\{B(p, l, q): p \geq 3, q \geq 3, l \geq 1\} \quad$ and $\mathrm{P}(l, p, q)=\{P(l, p, q): l, p, q \geq 1$ and at most one of them is 1$\}$. Denote $B(n)=\mathrm{B}(p, l, q) \cup \mathrm{P}(l, p, q)$.

Theorem 3.3 Let $G$ be a bicyclic graph. For $k=0,1, \ldots, n$, there exists some graph $H \in B(n)$ to satisfy the following inequality $c_{k}(G) \leq c_{k}(H)$.

Proof. Let $T_{i}$ be the tree attached at $u_{i}$. We can use Definition 2.2 to the vertex of $T_{i}$ which is the largest distance from the root $u_{i}$, as long as $T_{i}$ is not a path. By using Lemma 2.3, we increase Laplacian coefficients of $G$.

Assume that $G \in B_{n}^{\infty}$ has pendant path $P=a_{i} z_{1} z_{2} \cdots z_{r}$ attached at $a_{i},(2 \leq i \leq l-1)$. Let $G_{1}$ be the graph obtained from $G$ by removing the edge $a_{i} a_{i+1}$ and adding the edge $a_{i+1} z_{r}$. Obviously, we can have

$$
\begin{aligned}
& c_{0}(G)=c_{0}(H)=1, c_{1}(G)=c_{1}(H)= \\
& =2|E|, c_{n}(G)=c_{n}(H)=0, c_{n-1}(G)=c_{n-1}(H) .
\end{aligned}
$$

Let $F_{k}$ and $F_{k}^{H}$ be the sets of spanning forests of $G$ and $H$ with exactly $k$ components, respectively. Similar to the above prove, we can get the tree $T\left(T^{\prime}\right)$ on $G(H)$. By Lemma 2.10 and (2.1), it is easy to see that for every $0 \leq k \leq n$ holds $c_{k}(T) \leq c_{k}\left(T^{\prime}\right)$, with equality if and only if $k \in\{0,1, n-1, n\}$. We can get the same number of trees obtained from $G$ and $H$. According to Lemma 2.1, it is easy to see that for every $k=0,1, \ldots, n$, $c_{k}(G) \leq c_{k}(H)$, with equality if and only if $k \in\{0,1, n\}$.

Finally, we can apply $\eta_{1}$-transformation to the graph as long as it is not in $B(n)$. According to Theorem 3.2 and the above proof, we further increase its Laplacian coefficients. This means that the maximum Laplacian coefficients must be obtained from $B(n)$.

Definition 3.4 Let $G \in \mathrm{B}(p, l, q)$ and $p \geq 3, q \geq 3, l \geq 1$. Suppose that $u \in N_{C_{p}}\left(a_{1}\right), v \in V\left(C_{q}\right)$ and $d_{C_{q}}\left(a_{l}, v\right)=\left\lfloor\frac{q}{2}\right\rfloor$. Then $G_{2}=\eta_{2}(G, u, v)$ is a graph obtained by removing edge $u a_{1}$ and adding edge $u v$. We say that $G_{2}$ is a $\eta_{2}$ -transformation\} of $G$ (see Fig. 4, if $l=1$, see Fig. 5).

Theorem 3.5 Let $G \in \mathrm{B}(p, l, q)$ and $p \geq 3, q \geq 3, l \geq 1$. If $G_{2}=\eta_{2}(G, u, v)$, then for every $k=0,1, \ldots, n$, we have $c_{k}(G) \leq c_{k}\left(G_{2}\right)$.

Proof. Obviously, $c_{0}(G)=c_{0}\left(G_{2}\right)=1, c_{1}(G)=c_{1}\left(G_{2}\right)=$ If $=2|E|, c_{n}(G)=c_{n}\left(G_{2}\right)=0$

$q$ is an even number,
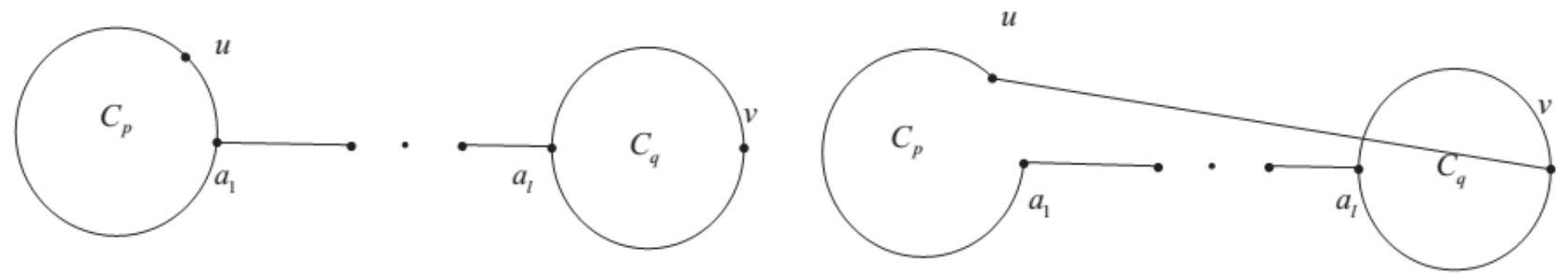

Figure 4. $\quad \eta_{2}$-transformation for $l>1$ 
$c_{n-1}(G)=n \tau(G)=n p q<n\left[(p+l-1) q+\left\lfloor\frac{q}{2}\right\rfloor\left\lfloor\frac{q}{2}\right\rfloor\right]=$ $=n \tau\left(G_{2}\right)=c_{n-1}\left(G_{2}\right)$

and if $q$ is an odd number,
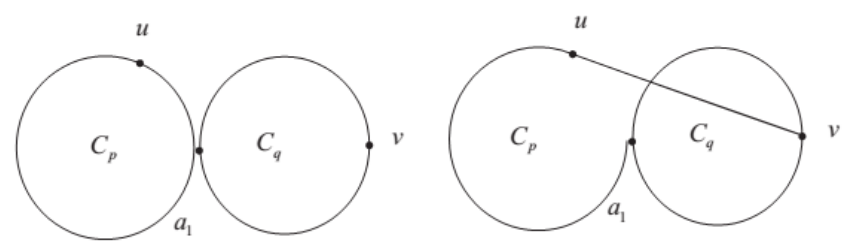

Figure 5. $\eta_{2}$-transformation for $l=1$

$c_{n-1}(G)=n \tau(G)=n p q<n\left[(p+l-1) q+\left\lfloor\frac{q}{2}\right\rfloor\left\lceil\frac{q}{2}\right\rceil\right]=$

$=n \tau\left(G_{2}\right)=c_{n-1}\left(G_{2}\right)$

Let $F_{k}$ and $F_{k}^{2}$ be the sets of spanning forests of $G$ and $G_{2}$ with exactly $k$ components, respectively. We distinguish the following two cases.

Case 1. $l=1$. According to the same reason, if we remove one of the edges in the cycle $C_{q}$, say $x y$, we can get the unicyclic graphs $U$ in $G$ and $U_{2}$ in $G_{2}$.

Obviously, by Definition 2.11, we know that $U_{2}=\gamma(U)$, and by Lemma 2.12, we can verify that $c_{k}\left(U_{2}\right) \geq c_{k}(U)$. By the above equation and Lemma 2.1, we have that for every $k=0,1,2, \ldots, n$, $c_{k}\left(G_{2}\right) \geq c_{k}(G)$, with equality if and only if $k \in\{0,1, n-1\}$.

Case 2. $l>1$. The next proof is the similar to that of Case 1. Then the conclusion of Theorem 3.5 holds.

According to the Theorem 3.5, we know that the graph with maximum Laplacian coefficients must be obtained from $\mathrm{P}(l, p, q)$.

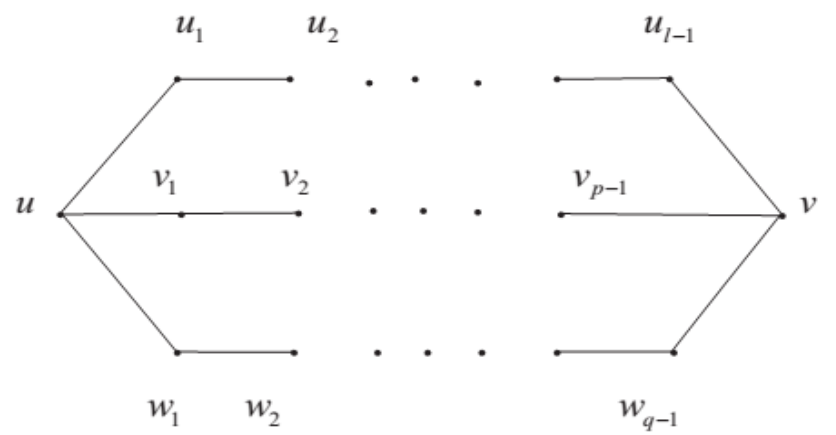

Definition 3.6 Suppose that $G \in \mathrm{P}(l, p, q)$. Without loss of generality, let $q \leq l \leq p$. Then $G_{3}=\eta_{3}(G)$ be a graph obtained by removing the edge $v u_{l-1}$ and adding the edge $v_{p-j} u_{l-1}$, where $j=\left\lceil\frac{p-q}{2}\right\rceil$, i.e. $q+j=p-j$ or $q+j=p-j-1$.

We say that $G_{3}$ is a $\eta_{3}$-transformation of $G$ (see Fig. $6)$.

Theorem 3.7 Let $G_{3}=\eta_{3}(G)$. Then for $0 \leq k \leq n$, $c_{k}(G) \leq c_{k}\left(G_{3}\right)$.

Proof. Obviously,

$c_{0}(G)=c_{0}\left(G_{3}\right)=1, c_{1}(G)=c_{1}\left(G_{3}\right)=$

$=2|E|, c_{n}(G)=c_{n}\left(G_{3}\right)=0$

$c_{n-1}(G)=n \tau(G)=n(l(p+q)+q-1)<$

$<n(l(p+q)+q+j-1)=n \tau\left(G_{3}\right)=c_{n-1}\left(G_{3}\right)$.

Case 1. If we remove the edge $u_{l-1} v$ in $G$ and $u_{l-1} v_{p-j}$ in $G_{3}$, we can get the unicyclic graphs $U_{1}$ and $U_{1}^{\prime}$, respectively. It is easy to see that $U_{1}=U_{1}^{\prime}$, therefore,

$$
c_{k}\left(U_{1}\right)=c_{k}\left(U_{1}^{\prime}\right)
$$

Case 2. If we remove the edge $u u_{1}$ in $G$ and $G_{3}$, we can get the unicyclic graphs $U_{2}$ and $U_{2}^{\prime}$, respectively. We have

$$
c_{k}\left(U_{2}\right)=c_{k}\left(U_{2}^{\prime}\right)
$$

Case 3. If we remove the edge $u v_{1}$ in $G$ and $u v_{1}$ in $G_{3}$, we can get the unicyclic graphs $U_{3}$ and $U_{3}^{\prime}$. By using Definition 2.11, $U_{3}^{\prime}=\gamma\left(U_{3}, v, v_{p-j}\right)$, and by Lemma 2.12,

$$
c_{k}\left(U_{3}\right) \leq c_{k}\left(U_{3}^{\prime}\right)
$$

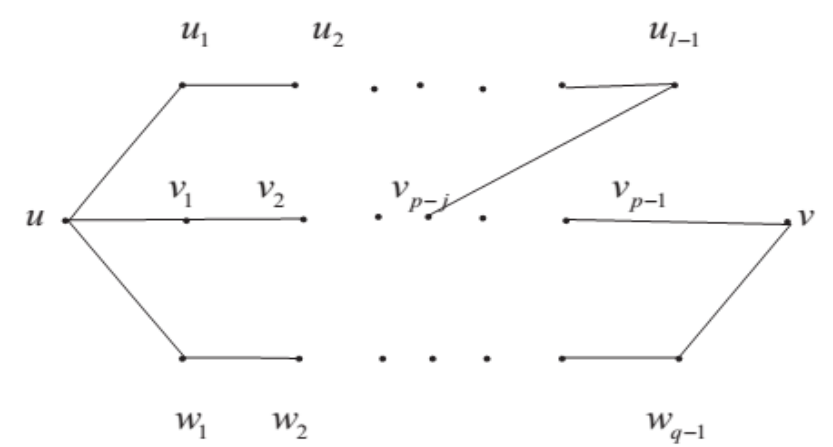

Figure 6. $\quad \eta_{3}$-transformation 
Case 4. If we remove the edge $v v_{p-1}$ in $G$ and $v v_{p-1}$ in $G_{3}$, we can get the unicyclic graphs $U_{4}$ and $U_{4}^{\prime}$. By Definition 2.11, $U_{4}^{\prime}=U_{4}-u_{l-1} v+u_{l-1} v_{p-j}$ $=\gamma\left(U_{4}, v, v_{p-j}\right)$, and according to Lemma 2.12, we have

$$
c_{k}\left(U_{4}\right) \leq c_{k}\left(U_{4}^{\prime}\right)
$$

Case 5. If we remove the edge $v_{p-i} v_{p-i-1},(i=1,2, \ldots, j-1) \quad$ in $\quad G \quad$ and $v_{p-j+i-1} v_{p-j+i},(i=1,2, \ldots, j-1)$ in $G_{3}$, we can get the unicyclic graphs $U_{5}(i)$ and $U_{5}^{\prime}(i)$. By Definition 2.11, there exists some vertex satisfied that $U_{5}^{\prime}(i)=\gamma\left(U_{5}(i)\right)$, and according to Lemma 2.12, we have

$$
c_{k}\left(U_{5}(i)\right) \leq c_{k}\left(U_{5}^{\prime}(i)\right) \quad(i=1,2, \ldots, j-1)
$$

Case 6. If we remove the edge $v_{i} v_{i+1},(i=1,2, \ldots, p-j-1) \quad$ in $\quad G \quad$ and $v_{i} v_{i+1},(i=1,2, \ldots, p-j-1)$ in $G_{3}$, we get the unicyclic graphs $U_{6}(i)$ and $U_{6}^{\prime}(i)$.

$$
c_{k}\left(U_{6}(i)\right) \leq c_{k}\left(U_{6}^{\prime}(i)\right) \quad(i=1,2, \ldots, p-j-1)
$$

Case 7. If we remove the edge $u_{i} u_{i+1},(i=1,2, \ldots, l-2) \quad$ in $\quad G \quad$ and $u_{i} u_{i+1},(i=1,2, \ldots, l-2)$ in $G_{3}$, we get the unicyclic graphs $U_{7}(i)$ and $U_{7}^{\prime}(i)$.

$$
c_{k}\left(U_{7}(i)\right) \leq c_{k}\left(U_{7}^{\prime}(i)\right) \quad(i=1,2, \ldots, l-2)
$$

According to (1)-(7) and Lemma 2.1, we have that $c_{k}(G) \leq c_{k}\left(G_{3}\right) \quad$ where $k=0,1, \ldots, n$. Then the conclusion of Theorem 3.7 holds.

Theorem 3.8 Let $G$ be a connected bicyclic graph on $n$ vertices and $k$ be an integer, $0 \leq k \leq n$. Let $t=n+1(\bmod 3)$,

(1) If $t=0$, then

$c_{k}(G) \leq c_{k}\left(P\left(\left\lceil\frac{n+1}{3}\right\rceil,\left\lceil\frac{n+1}{3}\right\rceil,\left\lceil\frac{n+1}{3}\right\rceil\right)\right)$;

(2) If $t=1$, then

$c_{k}(G) \leq c_{k}\left(P\left(\left\lceil\frac{n+1}{3}\right\rceil,\left\lfloor\frac{n+1}{3}\right\rfloor,\left\lceil\frac{n+1}{3}\right\rceil\right)\right)$;

(3) If $t=2$, then

$c_{k}(G) \leq c_{k}\left(P\left(\left\lfloor\frac{n+1}{3}\right\rfloor,\left\lfloor\frac{n+1}{3}\right\rfloor,\left\lceil\frac{n+1}{3}\right\rceil\right)\right)$.

Proof. Applying to Theorems 3.2, 3.3, and 3.5, we only need to prove that when $G \in \mathrm{P}(l, p, q)$, the result is correct. Without loss of generality, let $q \leq l \leq p$.

When $t=0$, according to Theorem 3.7, we can apply $\eta_{3}$-transformation as long as it is not the $P\left(\left\lceil\frac{n+1}{3}\right\rceil,\left\lceil\frac{n+1}{3}\right\rceil,\left\lceil\frac{n+1}{3}\right\rceil\right)$. Obviously, we increase its Laplacian coefficients. When $t=1$ and $t=2$, the proof is similar to $t=0$, we omit it.

\subsection{Laplacian-like Energy of Bicyclic Graphs}

Let $G$ be a graph. The Laplacian-like energy $(L E L)$ of graph $G$ is defined as follows: $\operatorname{LEL}(G)=\sum_{k=1}^{n-1} \sqrt{\mu(k)}$, where $\mu_{1} \geq \mu_{2} \geq \cdots \geq \mu_{n}=0$ are the Laplacian eigenvalues of $G$. Stevanovic in [7] presented a connection between $L E L$ and Laplacian coefficients.

Theorem 4.1. Let $G$ and $H$ be two graphs with $n$ vertices. If $c_{k}(G) \leq c_{k}(H)$ for $k=1,2, \cdots, n-1$, then $L E L(G) \leq L E L(H)$. Furthermore, if a strict inequality $c_{k}(G)<c_{k}(H)$ holds for some $1 \leq k \leq n-1$, then $\operatorname{LEL}(G)<\operatorname{LEL}(H)$.

Corollary 4.2. Let $G$ be a connected bicyclic graph with $n$ vertices. Then let $t=n+1(\bmod 3)$,

(1) If $t=0$ and $G \nsucceq P\left(\left[\frac{n+1}{3}\right\rceil,\left[\frac{n+1}{3}\right],\left\lceil\frac{n+1}{3}\right\rceil\right)$, then $L E L(G)<L E L\left(P\left(\left[\frac{n+1}{3}\right\rceil,\left\lceil\frac{n+1}{3}\right\rceil,\left\lceil\frac{n+1}{3}\right\rceil\right)\right) ;$

(2) If $t=1$ and $\left.G \nsucceq P\left(\frac{n+1}{3}\right\rceil,\left[\frac{n+1}{3}\right],\left\lceil\frac{n+1}{3}\right\rceil\right)$, then $\operatorname{LEL}(G)<\operatorname{LEL}\left(P\left(\left\lceil\frac{n+1}{3}\right\rceil,\left\lfloor\frac{n+1}{3}\right\rfloor,\left\lceil\frac{n+1}{3}\right\rceil\right)\right) ;$

(3) If $t=2$ and $G \supsetneqq P\left(\left[\frac{n+1}{3}\right\rceil,\left[\frac{n+1}{3}\right\rceil,\left\{\frac{n+1}{3}\right\rfloor\right)$, then $\operatorname{LEL}(G)<\operatorname{LEL}\left(P\left(\left\lceil\frac{n+1}{3}\right\rceil,\left\lceil\frac{n+1}{3}\right\rceil,\left\lfloor\frac{n+1}{3}\right\rfloor\right)\right.$.

\section{Acknowledgements}

The research has been supported by the Fundamental Research Funds for the Central Universities (No. 15CX02082A) and by NSF of China (Nos. 11371372, 61201455).

\section{REFERENCES}

[1] R. Merris, A survey of graph Laplacians, Linear and Multilinear Algebra 39(1995)19-31. 
[2] B. Mohar, On the Laplacian coefficients of acyclic graphs, Linear Algebra Appl. 722(2007)736-741.

[3] D. Stevanovic, A. Ilic, On the Laplacian coefficients of unicyclic graphs, Linear Algebra Appl. 430(2009)2290-2300.

[4] C. X. He, H. Y. Shan, On the Laplacian coefficients of bicyclic graphs, Discrete Math. 310(2010)3404-3412.

[5] X. Y. Pai, S. Y. Liu, J. M. Guo, On the Laplacian coefficients of tricyclic graphs, J. Math. Anal. Appl. 405(2013)200-208.

[6] A. K. Kelmans, V. M. Chelnokov, A certain polynomial of a graph and graphs with extremal number of trees, J.Combin. Theory, Ser. B.

[7] D. Stevanovic, Laplacian-like energy of trees, MATCH Commun. Math. Comput. Chem. 61(2009)407-417.
[8] A. Ilic, On the ordering of trees by the Laplacian coefficients, Linear Algebra Appl. 431(2009)2203-2212.

[9] A. Ilic, M. Ilic, Laplacian coefficients of trees with given number of leaves or vertices of degree two, Linear Algebra Appl. 431(2009)2195-2202.

[10] B. Zhou, I. Gutman, A connection between ordinary and Laplacian spectra of bipartite graphs, Linear and Multilinear Algebra 56(2008)305-310.

[11] S. W. Tan, Q. L. Wang, On the Laplacian coefficients of graphs under some trandformations, Linear Algebra Appl. 439(2013)2746-2761. 